\title{
Selective Handover Technique on Multihomed Mobile Network Environment*
}

\author{
Kiyong Park, Sunyong Han ${ }^{* *}$, and Jungwook Song \\ Computer and Information Communication Department, \\ Kon-kuk University, Seoul, Korea \\ kypark@cclab.konkuk.ac.kr, syhan@konkuk.ac.kr, \\ swoogi@konkuk.ac.kr
}

\begin{abstract}
Mobile Networks could have multiple wireless network interfaces due to various wireless technologies provided by many ISPs. Recently, those issues of the multiple connections, Multihomed Mobile Network, were highlighted in IETF NEMO WG. However, considering multiple connections has some tradeoffs between efficiency and fault tolerance, since the network must handle the status of all connections. Moreover, current NEMO basic support architecture does not have functions to handle those multiple connections when it encounters multiple new networks on new location. This article suggests a RA Message selection technique to support efficient Multihomed Mobile Network on NEMO basic support architecture even in case of the nested NEMO environment.
\end{abstract}

\section{Introduction}

Recently, to support Network Mobility [1], NEMO basic support architecture (NEMO BSA) [2], based on bi-directional tunnels [3] was selected as one of RFCs and its related techniques [4] are very similar to Mobile IP [5]. For these reasons, NEMO BSA has some critical problems such as route optimization problem called Pinball Route [6]. Meanwhile, the Mobile Router (MR) can receive several different Router Advertisement (RA) Messages in the new wireless network that the MR has moved. In the structure of NEMO BSA, because the MR does not have any functions to distinguish each of the RA messages except for Mobile Network Prefixes (MNP), the MR processes its handoff instantly whenever it receives a new RA message. In other words, if there are several RA messages that are being advertised, the MR might not know which RA message from each network is adequate and will handover whenever it detects different RA message. Hence, we could consider the NEMO Multihoming model [7] to support multiple connections on new network. However, previous NEMO BSA does not support multiple CoA registration on Home Agent [8]. In addition, one of the connections to a more deeply nested network could be of no use because of pinball route problem. Therefore, the MR must have some functions that select appropriate network and manage the connections.

In our paper, we are to propose a RA Message selection scheme for NEMO to prevent frequent handovers among new networks and to maintain an appropriate

\footnotetext{
"This research was supported by Seoul Research and Development program

** Corresponding author.
} 
connection even in the nested-NEMO [9] case. Chapter 2 describes related researches and current problems; chapter 3 explains entire architecture with detailed extensions; chapter 4 shows simulation results. Finally, the conclusion was described in chapter 5 .

\section{Related Researches}

\subsection{Multihoming in NEMO and Nested NEMO Model}

As said in [7], Mobile networks are typically connected by means of wireless and less reliable links; there could also be many nodes behind the MR. A loss of connectivity or a failure to connect to the Internet has a more significant impact than for a single node. Real-life scenarios as illustrated in [10] demonstrate that providing a permanent access to mobile networks such as vehicles typically require the use of several interfaces and technologies since the mobile network may be moving in distant geographical locations where different access technologies are provided and governed by distinct access control policies.

To support efficient handover in nested-NEMO model in terms of multihoming, previous research [11] considered the nested depth of mobile network. In this article, the nested depth of the mobile router is the most important factor of making a decision to select its parent-MR [9] in nested and NEMO multihoming model.

\subsection{Pinball Route Problem on NEMO Basic Support Architecture}

As we mentioned previously, there are bidirectional tunnels between a MR and its respective HA. These tunnels are used for forwarding data from CN [9] to MNN [9] behind the MR via the HA. Because all MNNs are using their addresses formed up from the home network prefix of the MR, the route to MNNs can not be on native route if the MR is not on the home network. Moreover, in assumption that the MR takes multiply nested-NEMO as its parent-NEMO, it causes a serious problem in the route path, called Pinball Route Problem. In this situation, the newly formed MR's CoA from its parent-MR is no more natively routable. Therefore n-times tunnel is indispensable for $\mathrm{CN}$ to reach MNNs under n-times nested-NEMO.

\subsection{Summary of Current Problems}

As previously mentioned, it can be supposed that NEMO multihoming model is applied to all MRs retaining the multiple wireless network interfaces with different technologies. Unfortunately, RA messages from one of MRs which has been nested and whose root-MR [9] has already lost its connection to the Internet can be advertised. We call this kind of MR 'Disabled MR' (D-MR). In this case, regarding only the depth of the nested-NEMO to determine the parent-MR among multiple parent-MRs is not sufficient. The worst case is that if the D-MR's signal strength is greater than the previous connection of a moving MR and each MR exchanges their RA messages simultaneously, the moving MR will form up its new connection with the D-MR's I-face [9] and the D-MR will form up its E-face [9] connection with the moving MR's I-face. We call this case 'Cross Registration in NEMO'. This is a critical problem on NEMO environment. Fig 1 shows these problems. 


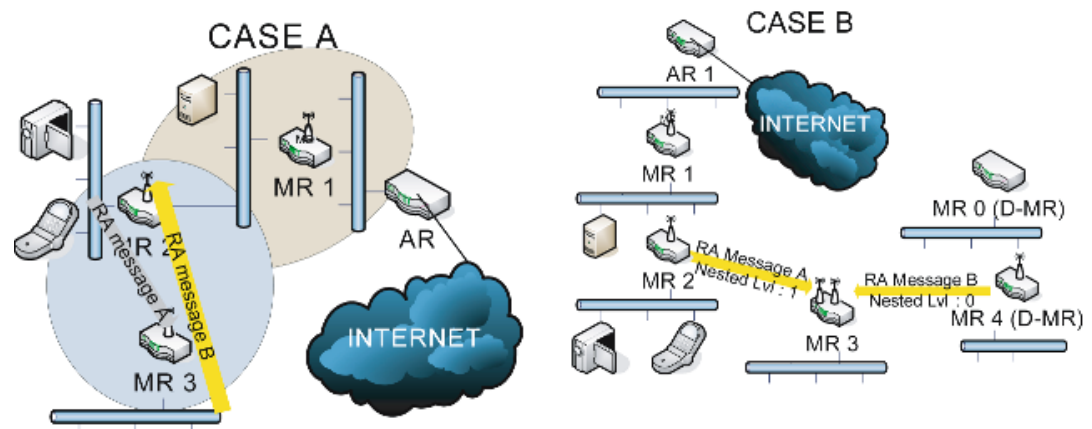

Fig. 1. Current Problems on Multihoming in nested Mobile Networking

Case A in Fig 1 shows 'Cross Registration' and Case B shows RA message comprehension using only the depth of nested-NEMO in RA message. However, in Case B, as you can see, MR3 can handover to MR4, which is D-MR because of lower depth value. Thus, that handover is of no use. As a result, more detailed information such as RTT value needs to be added for the sake of selecting appropriate RA message among multiple parent-MRs due to Pinball Route Problem.

In addition, the MR must register multiple CoAs to its respective HA. However, NEMO BSA does not have any capabilities to update the Binding List (BL) of the HA with multiple CoAs per HoA. Nevertheless, if there are multiple CoAs with different MNPs per HoA in the BL of the HA then it must monitor the status of all connections to CoAs due to some unexpected failures of the tunnels to those CoAs. These should cause huge workload on the HA.

\section{Architecture}

\subsection{Basic Concepts}

First of all, we must comprehend and separate appropriate one from various RA messages generated and advertised from parent-MRs. that means the MR in the intersection of the parent-MRs must select the best one for handover. Afterwards, the MR must select better one than previous selected or ignore recent one. To do so, we extended the nested depth of mobile network [11] as Nested Level (NL) and defined two more system values named Current Outgoing Link Status (COLS) and HA-RTT. The COLS implies whether the current connection to the Internet is available. In fact, current uplink status might already exist in each system. Therefore the system simply updates it. Finally, HA-RTT value between a MR and its respective HA is stored and updated periodically. And RA message is extended to store these three factors.

In the case of COLS, simply, if the uplink is working properly, COLS must be set to 1 or set to 0 . In the case of NL, the original RA message generated from AR means 'not nested'. If the MR receives a RA message without any extension part for above three factors, it can send its RA message to its local network with NL ' 1 ' instead of ' 0 ' in [11] and sub-MRs [9] behind the MR can understand that they are located under 1-time nested-NEMO. If the MR uses one of the RA messages from one of nested-NEMO, the 
NL is increased 1 more than as many as the RA message contains and stored in the system. Afterwards, only the NL is updated by the same RA messages without handover. However, if the NL was 0 , the MR must keep 0 without increasing and use it in its RA message. In addition, if the MR lost its connection, the NL values of the MR must be initiated. In other words, in our architecture, if COLS was set to 0 , the NL must be set to 0 and advertised instantly. This means that even the root-MR is not connected to the Internet and this is for announcing that the handover to this kind of NEMO is of no use. Finally, it is clear that HA-RTT value contains even whole workload to reach the respective HA including Pinball Route overload.

In network layer, multiple RA messages with different MNPs will be processed transparently by kernel functions such as 'ndisc_discovery' in IPv6 module in spite of multiple network interfaces which have different L2 technologies. For these reasons, using COLS, NL and HA-RTT are the main factors to decide the most appropriate RA message among different RA messages and some evaluation using these three factors was added in our scheme. It will be explained later. However, if the MR has only one E-face and it moved to the parent-MR which has 0 as NL or COLS by L2 process, it can not help turning into a D-MR.

\subsection{Operation Overview}

Fig. 2 shows how the RA messages that include newly defined three factors explained in previous chapter are sent to designated MR which is moving into new location.
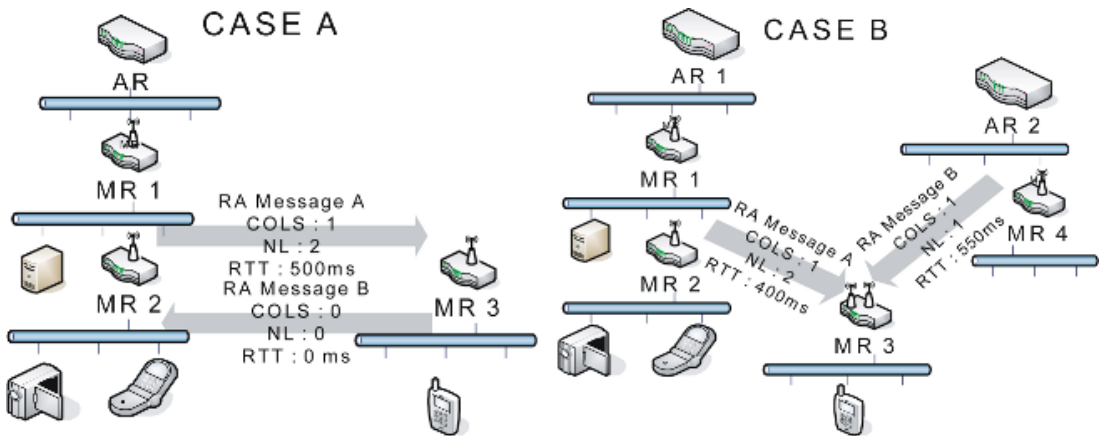

Fig. 2. Route Advertisement Message Flows

Case A is explaining that moving MR3 is approaching MR2 without its own connection to the Internet. So, MR3 is about to listen to the RA message from MR2 and MR2 is about to listen to it from MR3. Case B is that MR3 is approaching the range of the intersection between MR2 and MR4. RA message A was generated from MR2 and B is from MR4. Although, in the CASE B, we didn't draw the RA message generated from MR3, it is fully same RA message within CASE A.

First of all, in our architecture, MR will ignore all RA messages representing 0 as COLS. In such case, all MR would not start handover and it is clear that the NL is set to 0. Additionally, if the NL was set to 0 in the RA message in spite the COLS was set to 1 , it means that the mobile network is nested and there is no point of attachment to the Internet even in the parent-MRs. Therefore, the MR that heard this kind of RA 
messages discards it. In CASE A, because the RA message B includes 0 as COLS, MR2 would not process to the RA message B but MR3 uses the RA message A from MR2. In addition, if the RA message does not contain NL, COLS and RTT fields, it means the RA message is from the AR. In this case, the MR3 must select the RA message without any complexity if it is not currently connected to another AR.

In CASE B, the MR3 is on the intersection of two networks which are advertising their own RA messages and we can imagine that the MR3 would process the RA message A first. After a moment, in this case, the MR3 will change the connection to the MR4 as soon as it receives the RA message B despite the handover to the MR2 because the evaluation using NL, COLS and RTT of the RA Message B is better than the value of the RA Message A. in the contrary, if MR3 received the RA message B first, the RA message A is discarded.

There is one more case that we didn't describe in the figure. Let's suppose a group of several NEMOs nested hierarchically and the root-MR has no connectivity to the Internet is moving and one (named nMR) of the MRs in that group, but not root-MR, is approaching the MR4 in CASE B in Fig 2. In this situation, the MR4 receives the RA message including 1 as COLS and 0 as NL from nMR. As we explained before, the MR4 would not accept the RA message because of the NL in spite of the COLS. In the contrary, the RA message B from MR4 affects the nMR and the group of nested NEMOs approaching the MR4 will be rearranged in nested status because the nMR becomes the root-MR in that group.

\subsection{Extensions and Characteristics}

As mentioned in the basic concept, we defined several new functions in NEMO BSA. Those extensions summarized below. First, the RA message extensions are:

(1) Nested Level Count Field ( 2 bytes)

- $\quad$ Extended part of Valid Options field to store the Nested Level

(2) RA Message Bit, named 'L'

- One of the reserved bits in RA message format

- If it was checked, COLS means 1 or 0

(3) RTT time between the parent-MR and its respective HA (4 bytes)

The most important extension is the functions of MR. We stated those below.

(1) Three system variables to store COLS, NL and RTT

(2) Parsing above three factors from the RA messages

(3) Periodically, the MR checks its COLS : 1 as 'on' or 0 as 'off', RTT and update periodically

- $\quad$ Additionally, if COLS system variable is set to 0 , NL must be set to 0

(4) Store NL system value as 1 increased comparing to incoming RA message

(5) the NL value of 0 must be kept and stored in the MR and used in its RA message for its sub-MRs

(6) Ignore or accept the RA message by some evaluation function using COLS, NL and RTT

(7) If the same RA message received, only updates the NL from it. 
As presented in the above, our mechanism eliminates the complexities of handoffs on multihomed-NEMO environment, in our method,

A. There are no handover storms and cross registrations between MRs

B. The HA need not to manage which CoA is the best one or unreachable, that means our method could minimize workloads of HA for the MR's multiple network interfaces

C. Supports Load Balance due to the appropriate parent-MR selection at that time

D. Our mechanism minimizes the delay caused by Pinball Route Problem in NEMO BSA

\section{Performance Evaluation}

In real world, because we couldn't make the test suit for huge nested and mobile networks, we used OMNet++ [12], one of the network simulators, to evaluate our model. We supposed three nested mobile networks which have different NL each other are forming up their intersection on the specific location geometrically and a moving MR, named oMR, located on the intersection. Finally, NEMO BSA and our architecture are compared in the point of the processing and delay time in communicating between $\mathrm{CN}$ and oMR.

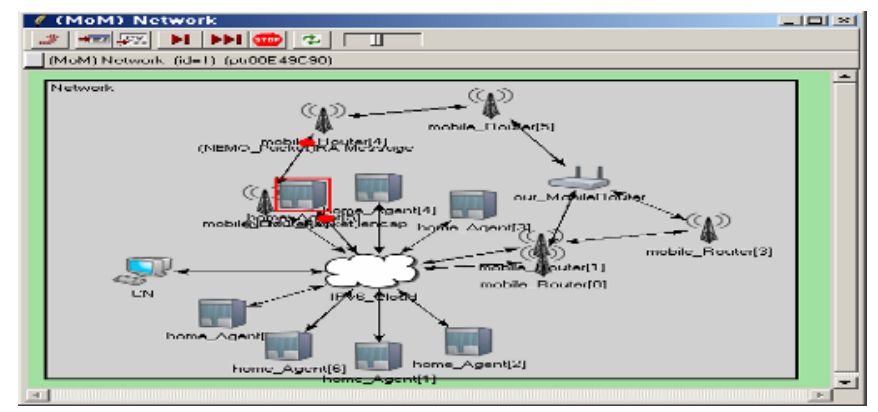

Fig. 3. The mobile networks to be simulated

In the Fig 3, MR3's NL is 2, MR5's NL is 3 and MR0's NL is 1. Each MR advertises its RA message with each of NL, COLS and RTT in each 1 second and the advertisement is started by MR5 first, MR3, MR0 and the oMR doesn't possess its own connectivity to the Internet. We calculated delay times of the packets from $\mathrm{CN}$ to oMR. We supposed 50 100ms delays to be routed in IPv6 Cloud, 10ms delays between sub-MR and parent-MR and each packet from $\mathrm{CN}$ to oMR is generated every 100ms. Fig 4 shows the result of our simulation and the total delay time from NEMO BSA takes much more than our architecture. In NEMO BSA, because oMR takes each RA message from MR3, MR5, MR0 by turn, the packet route is changed frequently. These make the packet delay unstable and taken more time. However, because our architecture uses only the most efficient RA message among them as Active CoA, thus the delay could be minimized. 

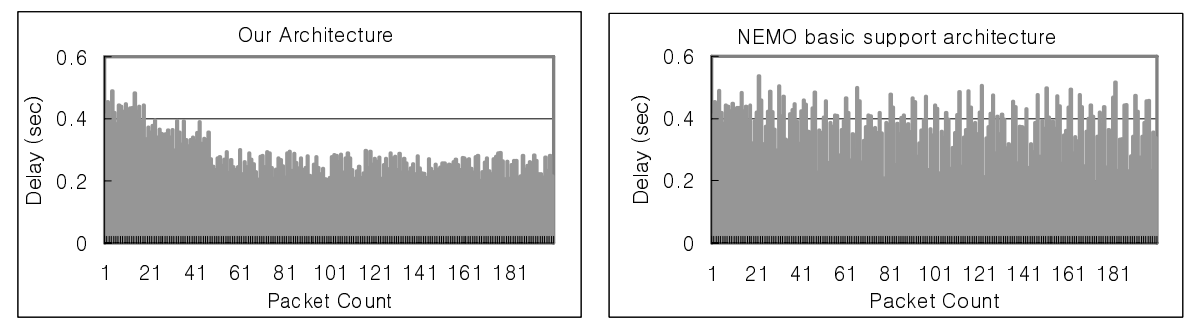

Fig. 4. Simulation results 1

Fig 5 shows the result of the case that only MR0 lost its connectivity to the Internet, that is Disabled MR, but advertising its RA to oMR. This evaluation was focused on the delay time whenever oMR sends its data to CN. In our architecture, MR0 was ignored due to COLS and NL, therefore, oMR choose MR3 as the parentMR and sent the information to its HA. However, in NEMO BSA, because oMR selected all RA messages from the parent-NEMOs like we simulated the case before, Clear and the most significant point of this case was that oMR couldn't send its data to the $\mathrm{CN}$ through MR0. Moreover, since NEMO BSA is suffered from binding update storms, total cost for the communication must be greater than this result. Note that delay 0 means loss of packets. Finally, the results show that our method is more stable and efficient than the handoff processes of NEMO BSA for the communications.
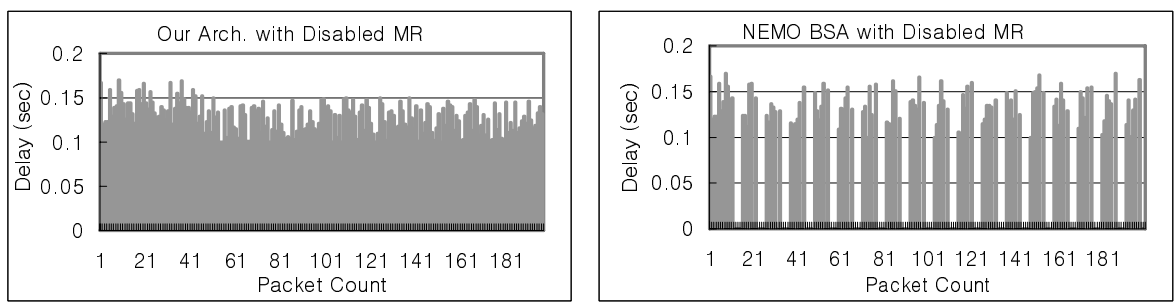

Fig. 5. Simulation results 2

Fig 6 shows how new MRs select their parent-MR among MR0, MR3 and MR5. in this situation, each MR calculates Workload with NL, RTT and 'weight value'. We evaluated like below. ('weight value' means tunnel process overhead and was temporarily supposed as 50)

$$
\text { Evaluation Result }=\mathrm{NL} *(\text { weight value })+\mathrm{RTT}
$$

These graphs mean each parent-MR's total Workload whenever new MR selects one as their parent-MR. As you can see, each Workload goes to around same value when new MRs are numerously increased. That means our technique is Loadbalanceable. 


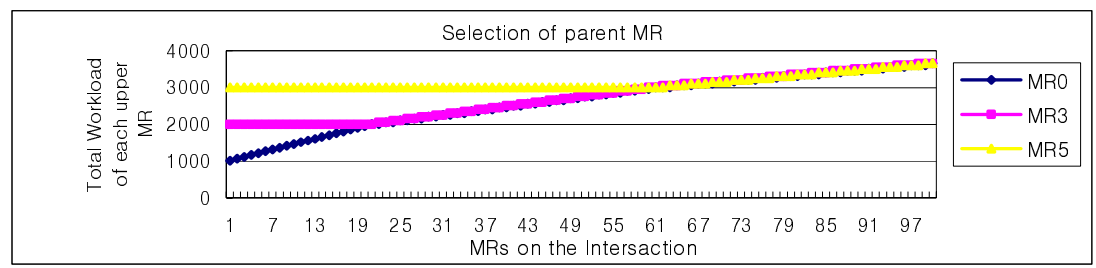

Fig. 6. Total Workload on MR0, MR3, MR5

\section{Conclusion}

In this paper, we examined and analyzed problems of NEMO multihoming model on NEMO BSA, and proposed new one that provides efficient and optimized RA message selection technique. With our architecture, we achieved optimal usage of multiple connections on NEMO multihoming model. In order to support our theory, we extended some functions of MR. Because this extension is based on NEMO BSA standard in terms of the functions and protocols; it does not result in much modification or loads to the network components. We also simulated some model to evaluate the performance of our approach. It showed that much lower delay for MNNs in a nested-NEMO to reach its correspondent nodes was taken, compared to NEMO BSA.

\section{References}

[1] T. Ernst, "Network Mobility Support Goals and Requirements", IETF Draft, 2005

[2] V. Devarapalli, R. Wakikawa, A. Petrescu, P. Thubert, "Network Mobility (NEMO) Basic Support Protocol", IETF RFC 3963, 2005.

[3] Conta, A. and S. Deering, "Generic Packet Tunneling in IPv6 Specification", RFC 2473, December 1998.

[4] J. Manner, M. Kojo, "Mobility Related Terminology", RFC3753, IETF, June 2004.

[5] D. Johnson, C. Perkins, Jari Arkko, "Mobility Support in IPv6", IETF RFC 3775, 2004.

[6] Thubert, P., and Molteni, M., "Taxonomy of Route Optimization Models in the NEMO Context", Internet Draft: draft-thubert-nemo-ro-taxonomy-00, Oct 2002

[7] C. Ng. "Analysis of Multihoming in Network Mobility Support", IETF Draft, 2005

[8] Ernst, T. and J. Charbon, "Multihoming with NEMO Basic Support", Proceedings First International Conference on Mobile Computing and Ubiquitous Networking (ICMU), January 2004.

[9] T. Ernst, "Network Mobility Support Terminology", IETF nemo Draft, Oct 2005

[10] Ernst, T., Montavont, N., Wakikawa, R., Paik, E., Ng, C., Kuladinithi, K., and T. Noel, "Goals and Benefits of Multihoming", draft-multihoming-generic-goals-and-benefits-02 (work in progress), October 2005

[11] Nicolas Montavont, "Multihoming in Nested Mobile Networking", International Symposium on Applications and the Internet Workshops, 2004

[12] OMNeT++ version 3.1, Home Page http://www.omnetpp.org 